\title{
EGFR tyrosine kinase inhibitors promote pro-caspase-8 dimerization that sensitizes cancer cells to DNA-damaging therapy
}

\author{
Yun-Tian Li ${ }^{1, *}$, Xiao-Jun Qian ${ }^{1,2, *}$, Yan Yu ${ }^{1, *}$, Zhen-Hua Li ${ }^{3}$, Rui-Yan Wu ${ }^{1}$, Jiao $\mathrm{Ji}^{1}$, \\ Lin Jiao ${ }^{1}$, Xuan Li ${ }^{1}$, Peng-Fei Kong ${ }^{1}$, Wen-Dan Chen ${ }^{1}$, Gong-Kan Feng ${ }^{1}$, Rong Deng ${ }^{1}$ \\ and Xiao-Feng Zhu ${ }^{1}$ \\ ${ }^{1}$ State Key Laboratory of Oncology in South China, Collaborative Innovation Center for Cancer Medicine, Cancer Center, Sun \\ Yat-sen University, Guangzhou, China \\ 2 Department of Oncology, Anhui Provincial Hospital, Affiliated to Anhui Medical University, Hefei, China \\ ${ }^{3}$ The School of Medicine, Jinan University, Guangzhou, China \\ * These authors have contributed equally to this work \\ Correspondence to: Xiao-Feng Zhu, email: zhuxfeng@mail.sysu.edu.cn \\ Keywords: EGFR inhibitors, doxorubicin, breast cancer, caspase-8, sequential application \\ Received: February 11,2015 Accepted: April 09, $2015 \quad$ Published: April 29, 2015
}

This is an open-access article distributed under the terms of the Creative Commons Attribution License, which permits unrestricted use, distribution, and reproduction in any medium, provided the original author and source are credited.

\section{ABSTRACT}

The combination of time and order-dependent chemotherapeutic strategies has demonstrated enhanced efficacy in killing cancer cells while minimizing adverse effects. However, the precise mechanism remains elusive. Our results showed that pre-treatment of MCF-7 and MDA-MB-468 cells with epidermal growth factor receptor (EGFR) tyrosine kinase inhibitor erlotinib or lapatinib significantly enhanced the cytotoxic effects of DNA-damaging agents compared to coadministration of the EGFR inhibitor and DNA-damaging agent. Sequential application of erlotinib and doxorubicin increased activated caspase- 8 by promoting pro-caspase- 8 homodimerization and autocatalytical cleavage, whereas coadministration did not. We found that EGFR inhibitors promoted pro-caspase-8 homodimerization by inhibiting ERK pathway signaling, while doxorubicin promoted it. Our data highlight that ERK has the potential to inhibit the formation of pro-caspase-8 homodimers by phosphorylating procaspase-8 at $\mathbf{S 3 8 7}$. In conclusion, the pretreatment of EGFR tyrosine kinase inhibitors promote pro-caspase-8 dimerization that sensitizes cancer cells to DNA-damaging agents. Our findings provide rationale for novel strategies for the implementation of combined targeted and cytotoxic chemotherapy within a new framework of time and order-dependent therapy.

\section{INTRODUCTION}

Over the past decades, several important, physiologic mechanisms of cell death have been described: (1) Apoptosis, a mechanism of programmed cellular death, involves two major pathways: the 'extrinsic' and 'intrinsic' pathway [1, 2]; (2) Autophagy, is known as a nonapoptotic model of cell suicide but the details regarding its underlying process remain controversial [3, 4]; (3) Necroptosis, like apoptosis and autophagy, is controlled by a regulated program but characterized microscopically by a necrotic phenotype $[5,6]$. Activation of any of the above pathways is a potentially catastrophic event for the cell and remains one of the mechanisms by which a malignant cell can kill itself in the presence of a drug [7]. A better understanding of the mechanisms by which anticancer drugs exert such effects is essential to improving the efficacy of combination therapies and limiting the likelihood of resistance development. Doxorubicin (DOX) is a major anthracycline chemotherapeutic agent used in the treatment of breast cancer despite dose-limiting adverse effects, such as cardiotoxicity, and the potential for facilitating the development of multidrug resistance $[8$, 9]. To address these limitations and improve its efficacy, 
DOX is often supplemented by combination with other chemotherapeutic agents [10]. However, this genotoxic combination induces the intrinsic apoptosis pathway through DNA damage additionally $[11,12]$. Thus, the combined use of chemotherapies with similar mechanisms of action has limited efficacy and can potentially facilitate the development of drug resistance.

Novel targeted therapies have shown considerable clinical efficacy with improvements in overall survival across a spectrum of human cancers [13-15]. The potential efficacy of a novel, combined therapeutic strategy utilizing tyrosine kinase inhibitors (TKIs) alongside cytotoxic chemotherapy has previously been explored in the treatment of breast cancer. However, EGFR inhibition in combination with genotoxic agents such as cisplatin have resulted in less than a $10 \%$ survival benefit [16]. Moreover, the addition of EGFR inhibitor cetuximab to carboplatin did not improve outcomes in a randomized phase II trial in triple negative breast cancer (TNBC) patients [17]. While these results are far from encouraging, experimental data indicate that time-staggered EGFR inhibition, as opposed to simultaneous co-administration, can dramatically sensitize a subset of triple-negative breast cancer cells to genotoxic drugs [18]. The same phenomenon has also been demonstrated in non-small cell lung carcinoma (NSCLC). In four randomized phase III trials [19, 20], while concurrent administration of erlotinib or gefitinib with standard platinum-doublet chemotherapy did not improve survival compared with chemotherapy alone, the sequential, staggered scheduling of erlotinib followed by cytotoxic chemotherapy led to a significant improvement in progression-free survival (PFS) in patients with advanced NSCLC, in the multicenter, randomized phase II First-Line Asian Sequential Tarceva and Chemotherapy Trial (FAST-ACT) [21]. Pre-clinical evidence indicates a potential antagonism that exists between the constituents of such combination therapies when they are administered simultaneously [22]. On the other hand, the molecular mechanism underlying the efficacy of sequential coadministration has not been elucidated.

It has been shown that erlotinib-dependent caspase- 8 activation occurs following DNA damage, which activates the intrinsic apoptotic pathway, but the underlying molecular mechanism remains elusive [18]. Caspase- 8 activation through dimerization is known to recruit oligomeric activation platforms that assemble subsequent to activation of the extrinsic pathway [23-25]. It is also well-established that caspase- 8 phosphorylation induces the formation of a stable, inactive cytosolic dimer, and this hypothesis has been proven through Lyn induced pro-caspase- 8 phosphorylation and dimerization [26]. The aim of our work was to identify cytosolic proteins affected by EGFR inhibition that promote caspase- 8 activation in a breast cancer model. We found that procaspase- 8 activation was induced by EGFR inhibitors, with subsequent activation of the downstream caspase- dependent pathways, including both the extrinsic and the intrinsic apoptotic cascades. Our findings demonstrate a potential mechanism underlying the efficacy of sequential scheduling of combined TKIs and genotoxic chemotherapy administration.

\section{RESULTS}

\section{Sequentially application of EGFR inhibitor followed by doxorubicin mediated cell death}

The growth inhibition of the EGFR inhibitors erlotinib and lapatinib in the breast cancer MCF-7 cells was examined. Surprisingly, both erlotinib and lapatinib did not yield an apparent effect on MCF-7 cell viability, even at $10 \mu \mathrm{M}$ concentration and $48 \mathrm{~h}$ incubation (Figure $1 \mathrm{~A}$ and $1 \mathrm{~B})$. This negligible inhibition indicates that MCF-7 cells exhibit strong resistance to EGFR inhibition. To improve the growth inhibition of MCF-7 cells, the time-staggered administration of EGFR inhibitors was conducted followed by DNA intercalating agent doxorubicin, which has been reported to dramatically inhibit a subset of triple-negative breast cancer cells. We found that $10 \mu \mathrm{M}$ doxorubicin could reduce cell proliferation by $30-50 \%$ (Figure 1C and 1D). Simultaneous co-administration showed no difference compared to doxorubicin alone. Interestingly, however, combinations in which EGFR inhibitors were administered $24 \mathrm{~h}$ prior to doxorubicin demonstrated a markedly enhanced inhibition of proliferation, with an inhibitory rate of $70-90 \%$. When the order of drug presentation was reversed-doxorubicin given before erlotinib - cell killing was not enhanced relative to treatment with doxorubicin alone (Figure 1C and 1D). We next studied cell death responses to the combination of erlotinib and doxorubicin by AnnexinVPI staining (Figure 1G). Consistent with the MTT assays, time and order-dependent combination of erlotinib and doxorubicin led to a markedly enchanced apoptotic rate. Our results are consistent with a prior study by Lee et al. [18].

Although EGFR is overexpressed in MDA-MB-468 cells, they were also insensitive to EGFR inhibition. To verify and validate the efficacy of the order and timing of drug administration in this model, the potential for synergistic killing was also explored following timestaggered pre-treatment with either erlotinib or lapatinib in combination with doxorubicin. As expected, pre-treatment with EGFR inhibitors could also sensitize the MDAMB-468 cells to doxorubicin (Figure 1E and 1F). 


\section{Sensitization to doxorubicin-induced cell death by pre-treatment with erlotinib is mediated via caspase-8}

We next sought to identify the molecular basis for the observed difference in therapeutic efficacy between simultaneous co-administration versus sequential dosing.
Activation of the NF- $\mathrm{kB}$ family of transcription factors is known to mediate cellular responses to DNA-damaging therapies; however, there was no apparent difference in the activation of the NF- $\kappa$ B pathway between these two groups (Figure 2A). As DNA damaging agents commonly induce apoptosis through activation of caspase-9, key mediator of the intrinsic apoptosis pathway, we investigated the role of caspase- 9 in the sequential,
A

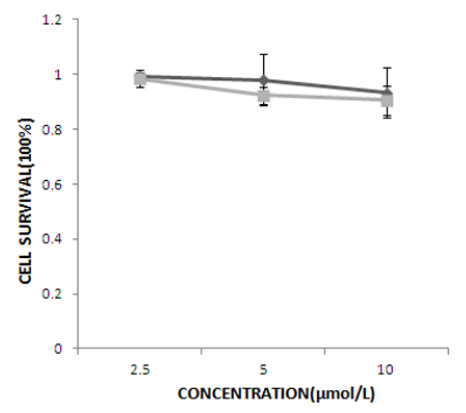

C

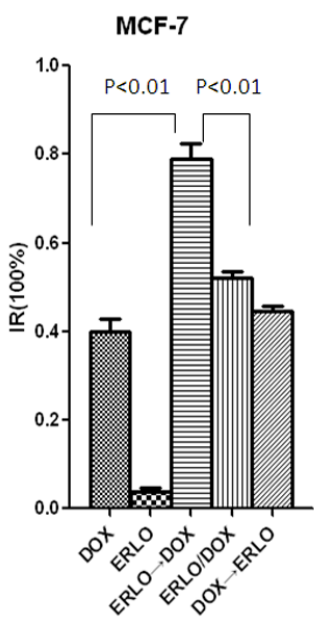

$24 \mathrm{H}$
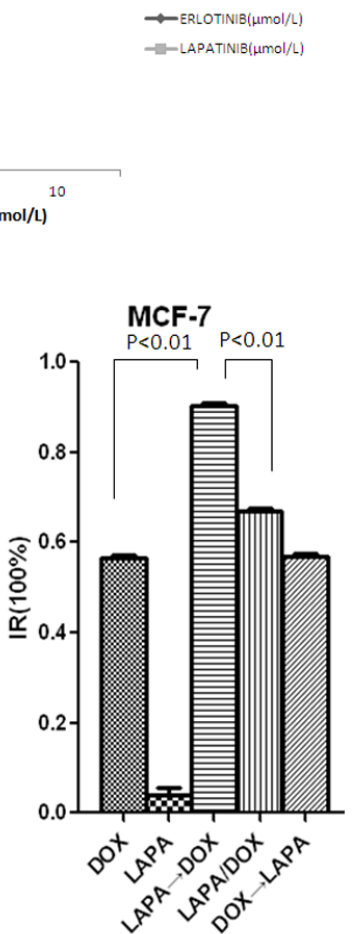
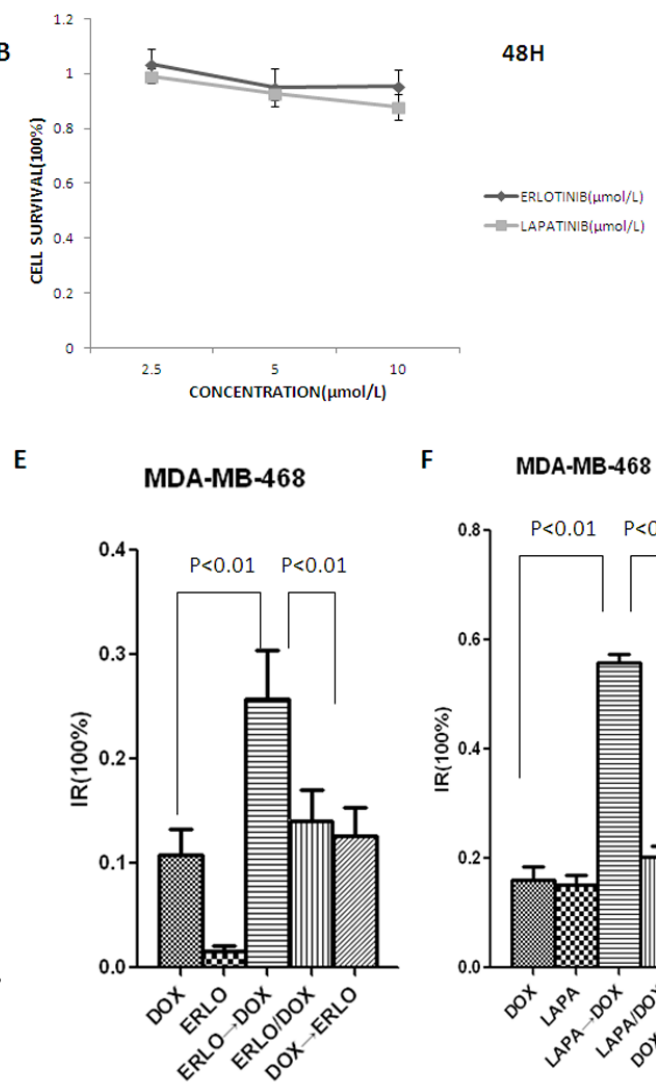

F

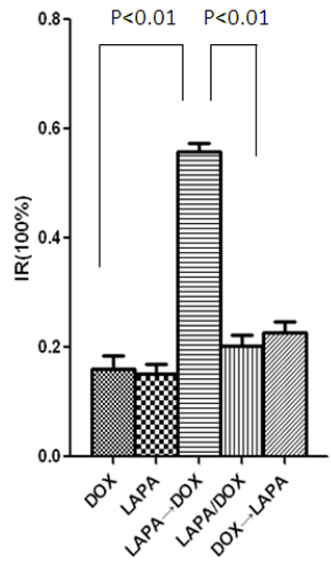

G
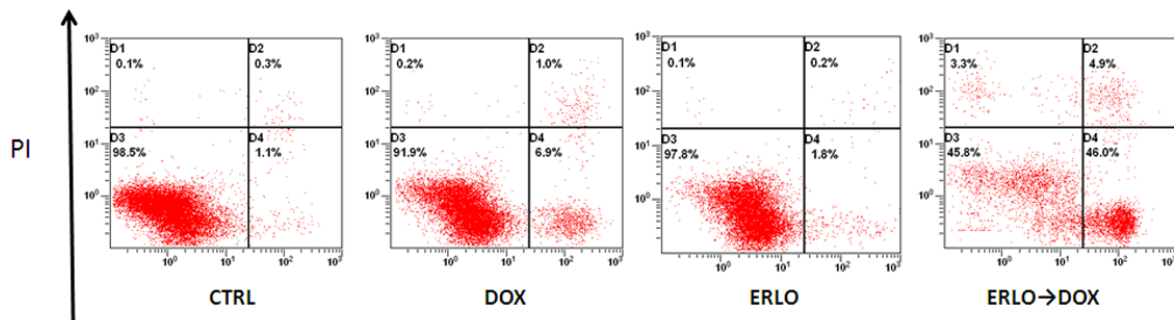

ERLO $\rightarrow$ DOX

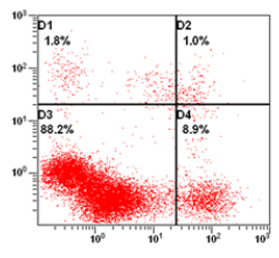

ERLO/DOX

Annexin-V

Figure 1: Sequential scheduling of EGFR inhibitors with doxorubicin in breast cancer cells. A, B. Inhibitory effects of erlotinib and lapatinib on MCF-7 cell proliferation. Cell growth was assessed using the MTT assay after treatment with erlotinib or lapatinib for 24hr or 48hr. C-F. Inhibitory effects of sequential combination of EGFR inhibitors with doxorubicin on MCF-7 and MDAMB-468 cells proliferation. ERLO $\rightarrow$ DOX or LAPA $\rightarrow$ DOX refer to erlotinib or lapatinib given $24 \mathrm{~h}$ before doxorubicin; DOX $\rightarrow$ ERLO or $\mathrm{DOX} \rightarrow \mathrm{LAPA}$ refer to doxorubicin given $4 \mathrm{~h}$ before erlotinib or lapatinib; ERLO/DOX or LAPA/DOX refers to erlotinib or lapatinib and doxorubicin added at the same time. For each combination, cell growth was assessed using the MTT assay made $12 \mathrm{hr}$ after the addition of doxorubicin. IR: inhibitory rate. The concentrations of erlotinib, lapatinib and doxorubicin were used at $10 \mu \mathrm{M}$, unless stated otherwise. G. Apoptosis induced by sequential combination of EGFR inhibitors with doxorubicin on MCF-7. ERLO $\rightarrow$ DOX refer to erlotinib given $24 \mathrm{~h}$ before doxorubicin; ERLO/DOX refers to erlotinib and doxorubicin added at the same time. For each combination, apoptosis rate was analyzed by the flow cytometry assay. 
combined administration of erlotinib and doxorubicin. Interestingly, although silencing of caspase- 9 by siRNA reduced the inhibitory rate of both simultaneous and sequential co-administration, the staggered, sequential treatment strategy was still effective (Figure 2B). Then the extrinsic apoptotic pathways were analyzed. The results of Western blotting revealed that pre-treatment with erlotinib followed by doxorubicin markedly increased apoptosis in MCF-7 cells compared with simultaneous co-administration, as evidenced by higher levels in cleaved PARP after sequential treatment (Figure 2C). Furthermore, levels of the activated form of caspase-8, intermediate fragments $\mathrm{p} 43 / \mathrm{p} 41$ and $\mathrm{p} 18$, were also increased in sequential dosing (Figure 2D). Based on these findings, the role of the initiator caspase of the extrinsic apoptotic pathways, caspase-8, was further investigated. Treatment of MCF-7 cells with a pan-caspase inhibitor, Z.VAD-FMK, significantly suppressed doxorubicin- induced cell death and attenuated the difference between simultaneous co-administration and sequential dosing (Figure 2E left). To confirm the involvement of caspase- 8 in erlotinib-mediated chemosensitization to doxorubicin, MCF-7 cells with silencing of caspase- 8 by siRNA was preformed. Consistent with the result of Z.VAD-FMK administration, silencing of caspase- 8 also significantly suppressed doxorubicin-induced cell death and attenuated the difference between co-administration and sequential dosing (Figure 2E right). These results indicated that pre-treatment with erlotinib sensitized MCF-7 cells to doxorubicin by inducing the caspase- 8 mediated extrinsic apoptotic pathway.
A

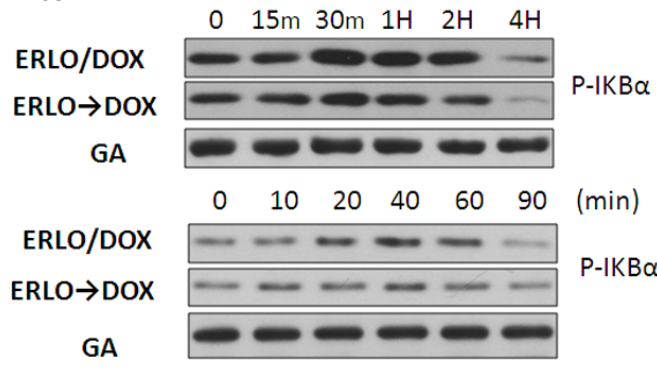

C $0 \quad 2 \mathrm{H} \quad 4 \mathrm{H} \quad 6 \mathrm{H} \quad 8 \mathrm{H} 10 \mathrm{H}$

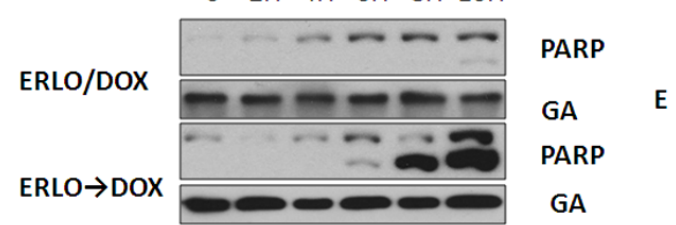

D

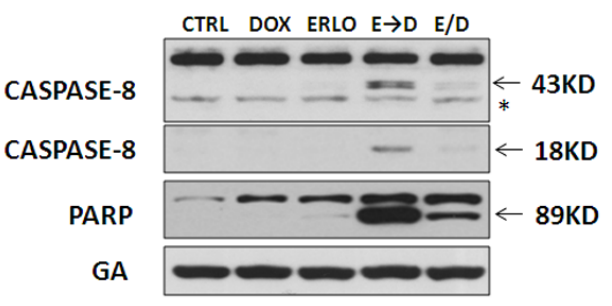

B
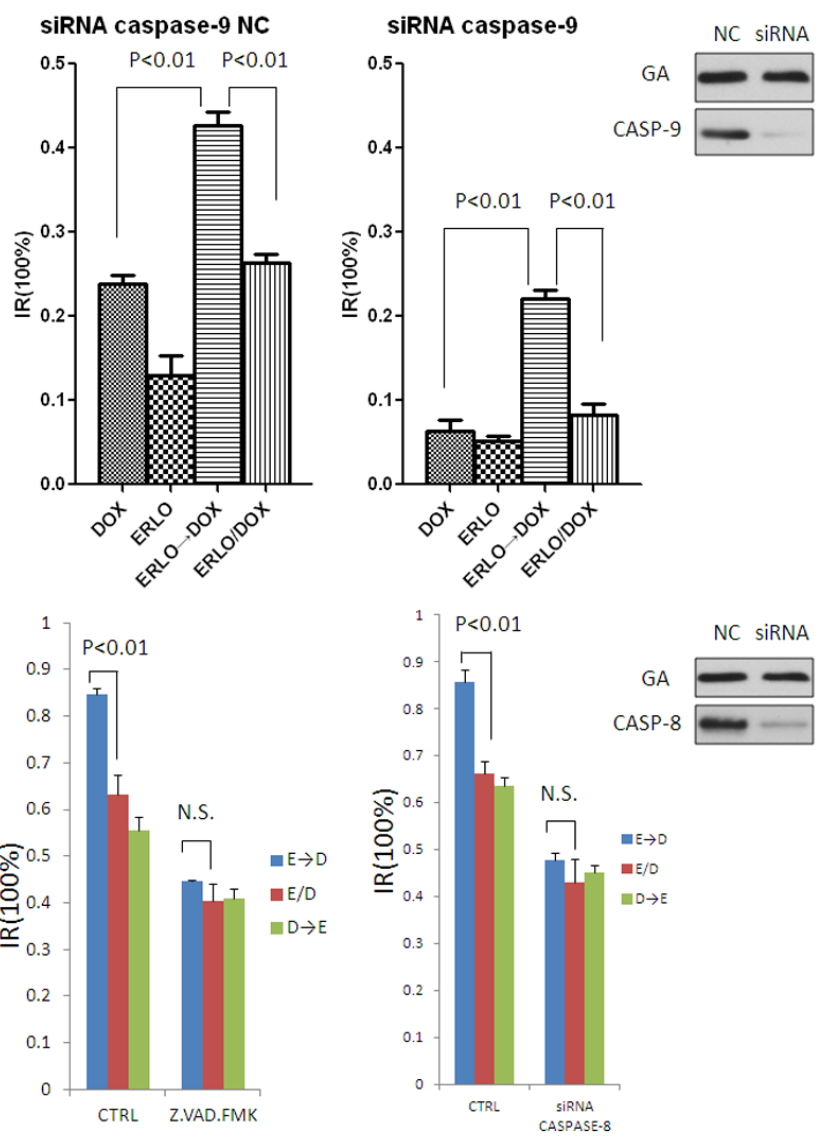

Figure 2: Sensitization for doxorubicin-induced cell death by pretreatment with erlotinib is mediated via caspase-8. A. Immunoblot analysis of phospho-IKB $\alpha$ in the ERLO $\rightarrow$ DOX and ERLO/DOX treated cells. MCF-7 cell extracts were collected at indicated times after the addition of doxorubicin. B. MTT assay assessing the sensitivity of DOX, ERLO $\rightarrow$ DOX and ERLO/DOX after knockdown of caspase-9 by siRNA. C. Immunoblot analysis of cleaved PARP in the ERLO $\rightarrow$ DOX and ERLO/DOX treated cells. MCF-7 cell extracts were collected at indicated times after the addition of doxorubicin. D. Immunoblot analysis of cleaved caspase- 8 and cleaved PARP in the DOX, ERLO, ERLO $\rightarrow$ DOX and ERLO/DOX treated cells. MCF-7 cell extracts were collected $6 \mathrm{~h}$ after the addition of doxorubicin. Asterisk indicates nonspecific binding of Abs. E. MTT assay assessing the sensitivity of ERLO $\rightarrow$ DOX, DOX $\rightarrow$ ERLO and ERLO/DOX after treated with caspase- 8 inhibitor Z.VAD.FMK $(20 \mu \mathrm{M})$ or knockdown of caspase- 8 by siRNA. N.S.: no significance. 


\section{Erlotinib promotes homodimerization}

pro-caspase-8

It is well known that activation of pro-caspase- 8 results from its homodimerization. We asked whether erlotinib may have an effect on the dimerization of procaspase- 8 in MCF-7 cells. To investigate this issue, the native-page was performed, and the band around the $130 \mathrm{KD}$ mark was regarded as a dimer of caspase-8, which has been previously reported and validated. MCF7 cells were treated with either erlotinib or doxorubicin respectively at indicated times. Interestingly, we found that erlotinib increased levels of this caspase- 8 dimer while doxorubicin reduced it (Figure 3A). To demonstrate that this was indeed a homodimer, a homolog of caspase-8, FLIP, which lacks a functional caspase domain but can form a heterodimer with caspase-8, was detected simultaneously. Indeed, FLIP was not detected at the same position around $130 \mathrm{KD}$, confirming that this dimer was the homodimer of caspase- 8 . Furthermore, we constitutively expressed FLAG-caspase- 8 and HAcaspase-8 in MCF-7 cells. Co-immunoprecipitation revealed that erlotinib increased the combination of Flagcaspase- 8 and HA-caspase-8, which was consistent with our native-page result (Figure 3B). Taken together, these data indicate that pre-treatment with erlotinib sensitized MCF-7 cells to doxorubicin by promoting pro-caspase- 8 homodimerization and activity.

\section{Erlotinib homodimerization by inhibiting pERK 1/2 \\ pro-caspase-8}

Dimerization plays a crucial role in the activity of many kinases, including Raf, CDK2 (cyclin-dependent kinase 2) and EGFR (epidermal growth factor receptor) [27]. However, dimerized conformational states are often stabilized by the appropriate phosphorylation of the appropriate residues. When pro-caspase- 8 homodimerization and activity was investigated, Lyn was demonstrated to phosphorylate pro-caspase- 8 at Tyr380, thereby inducing the formation of an inactive pro-caspase- 8 homodimer, while the mechanism inducing the formation of an active pro-caspase- 8 homodimer was unknown. Previous reports have suggested that caspase- 8 can be phosphorylated at its S387 residue and is a substrate of P-ERK [28]. Since P-ERK is a the major downstream effector of the EGFR pathway, which could also be downregulated by erlotinib-mediated EGFR inhibition, we asked whether erlotinib could induce procaspase- 8 homodimerization by attenuating pERK $1 / 2$ mediated phosphorylation of pro-caspase-8 at S387. To investigate this possibility, P-ERK was analyzed in MCF7 cells treated with either erlotinib or doxorubicin. As expected, without impacting the level of ERK, erlotinib downregulated P-ERK expression, while doxorubicin upregulated its expression (Figure 4A). Based on these findings, we hypothesized that ERK represents a key molecule in the phenomenon of sequential dosing. Thus, we replaced erlotinib with MEK inhibitors, AZD6244 and U0126, which have been reported to significantly reduce P-ERK levels. We found that ERK inhibition was sufficient to mimic the effects of erlotinib in sequential applications of anti-cancer drugs, as pre-treatment with AZD6244 or
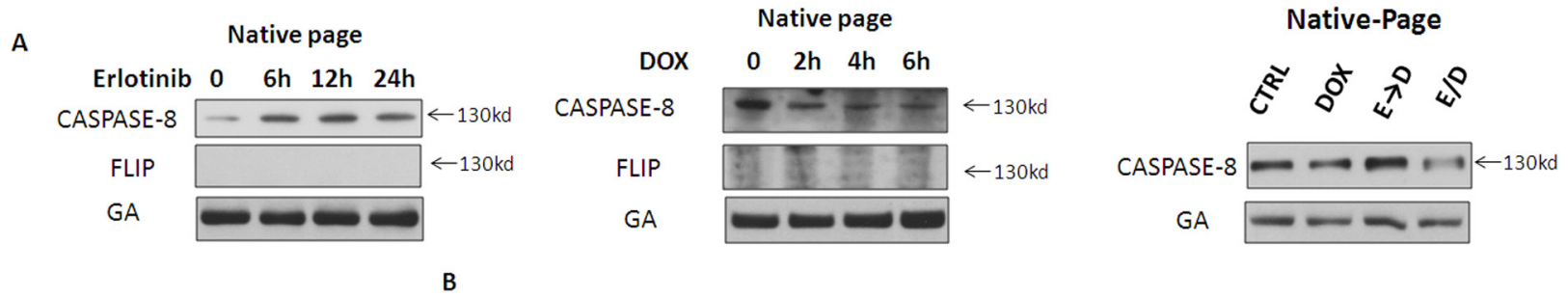

MCF-7 co-expression HA-CASPASE-8\&FLAG-CASPASE-8

WCL

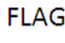

HA

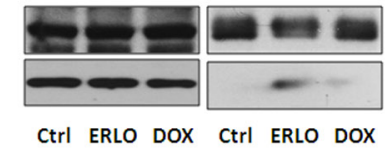

IP:FLAG

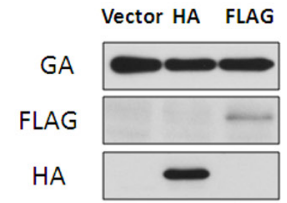

Figure 3: Erlotinib promotes pro-caspase-8 homodimerization. A. Cell lysates were analyzed by native-page Western blot to detect dimerization of pro-caspase-8 in MCF-7 cells. MCF-7 cells were treated with erlotinib, doxorubicin, ERLO $\rightarrow$ DOX or ERLO/DOX. For ERLO $\rightarrow$ DOX and ERLO/DOX, MCF-7 cell extracts were collected $6 \mathrm{~h}$ after the addition of doxorubicin. B. Coimmunoprecipitation assay to test for interactions between FLAG-caspase- 8 and HA-caspase-8 in MCF-7 cells by transfected Vector, HA-caspase-8 and FLAGcaspase-8. 
U0126 could also sensitize MCF-7 cells to doxorubicin treatment (Figure 4B). Furthermore, compared with negative controls, ERK knockdown by siRNA could also sensitize MCF-7 cells to doxorubicin treatment (Figure $4 C)$. These results provide further, adjunctive evidence that pre-treatment with erlotinib sensitized MCF-7 cells to doxorubicin by inhibiting downstream activation of P-ERK.

To demonstrate that P-ERK reduces pro-caspase- 8 homodimer, MCF-7 cells were treated with MEK inhibitors and siRNA knockdown and pro-caspase-8 homodimer levels were measured. Our data indicated that either MEK inhibition or siRNA knockdown could increase levels of this dimer (Figure 4D).

\section{Erlotinib induces pro-caspase-8 homodimerization by attenuating the phosphorylation of pro- caspase-8 at $\mathbf{S 3 8 7}$ mediated by pERK 1/2}

To examine whether phosphorylation of Ser387 by P-ERK is involved in erlotinib induced procaspase- 8 homodimerization, this site was replaced with a mutant, non-phosphorylatable amino acid (S387A). The S387A mutant significantly enhanced doxorubicin-induced cell death in MCF-7 cells and MDA-MB-468 cells (Figure
5A and 5B). Moreover, the non-phosphorylatable (S387A) caspase-8 attenuated the synergistic effects of the sequential application of erlotinib and doxorubicin compared to co-administration, suggesting that caspase-8 S387 plays a key role in the synergistic effects of sequential administration of erlotinib and doxorubicin (Figure 5A and 5B). These experiments allowed us to conclude that P-ERK impaired caspase- 8 activity in this system.

Next, we examined whether phosphorylation at the S387 residue by P-ERK modulates caspase- 8 dimerization. The native-page showed that MCF-7 cells with caspase- 8 S387A did not increase or even reduced dimerization with erlotinib or doxocubicin compared to the empty vector control (Figure 5C). Further experiment showed that the expression of caspase-8 S387A in MCF-7 cells eliminated the difference of pro-caspase- 8 autocatalytic activation with doxorubicin alone, simultaneous co-administration of erlotinib and doxorubin, as well as their sequential administration. In addition, apoptosis was activated equally among these three different treatment groups, as cleaved PARP showed no apparent difference (Figure 5D). Apoptosis analysis by Annexin V-FITC/PI staining showed that caspase-8 S387A in MCF-7 cells attenuated the enhanced apoptotic effect facilitated by the sequential application of erlotinib and doxorubicin (Figure 5E),

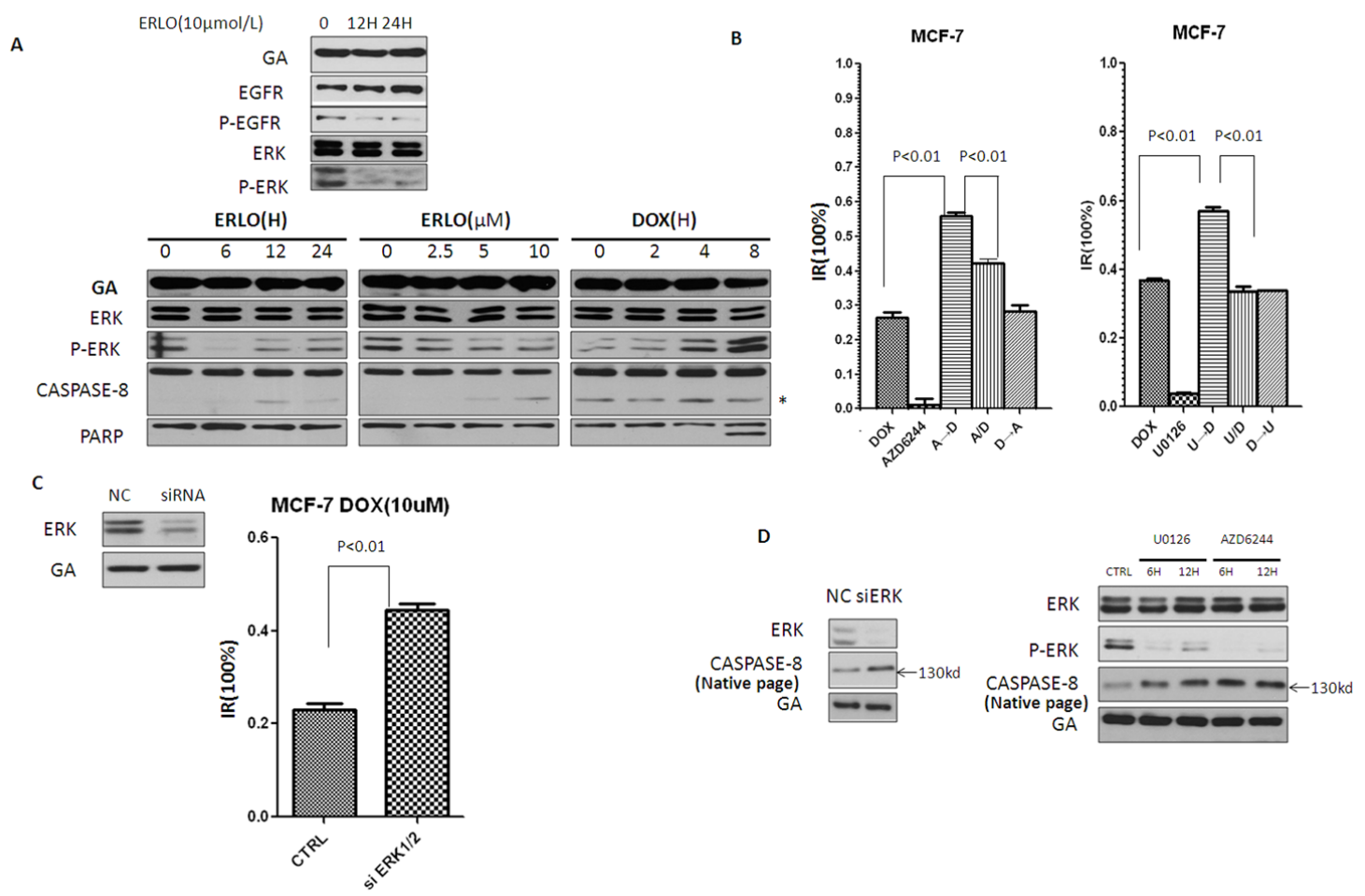

Figure 4: Erlotinib induces pro-caspase-8 homodimerization by inhibiting P-ERK. A. Immunoblot analysis of the level of P-ERK in MCF-7 cells treated with erlotinib and doxorubicin. Asterisk indicates nonspecific binding of Abs. B. MTT assay assessing sequential combination proliferation inhibitory of MEK inhibitors (AZD6244 and U0126) and doxorubicin in MCF-7 cells. MTT assay was made $12 \mathrm{hr}$ after the addition of doxorubicin. AZD6244 was treated at $5 \mu \mathrm{M}$, U0126 was treated at $10 \mu \mathrm{M}$, unless stated otherwise. C. Immunoblots of knock-down of in cells by siRNA. MTT assay assessing doxorubicin sensitivity of MCF-7 cells after ERK knockdown. D. MCF-7 cells were treated with MEK inhibitors and siRNA, pro-caspase8 homodimer was detected by Native-page Western blot analysis. 
which was consistent with MTT assay and Western blot analysis.

In general, these data indicate that phosphorylation of procaspase- 8 at $\mathrm{S} 387$ mediated by pERK 1/2 impairs erlotinib-induced procaspase- 8 homodimerization as well as caspase- 8 activation.

\section{DISCUSSION}

DNA damage [29], such as treatment with doxorubicin, leads to p53-mediated induction of Noxa and Puma and the subsequent induction of the intrinsic apoptosis pathway, which is mediated by caspase-9 [30]. In this study, we found that pre-treatment with erlotinib followed by doxorubicin could lead to activation of the extrinsic apoptotic pathway, which is mediated by caspase- 8 , while the simultaneous co-administration of these two distinct classes of anti-cancer agents showed no induction of extrinsic apoptosis. We hypothesize that the synergistic therapeutic effect of sequential administration of EGFR inhibitor followed by doxorubicin was related to the co-induction of the intrinsic and extrinsic apoptotic pathways.

The importance of the MAPK pathway [31]
A
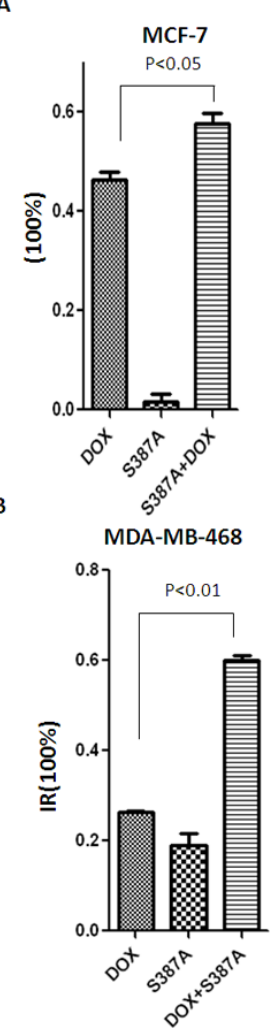

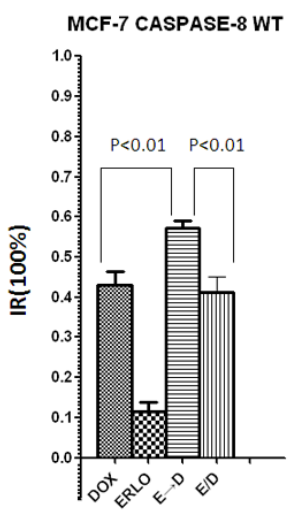

MDA-MB-468 WT

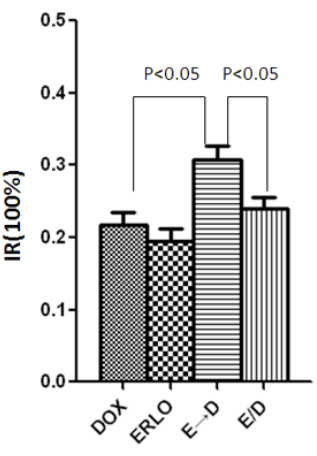

MCF-7 CASPASE-8 S387A
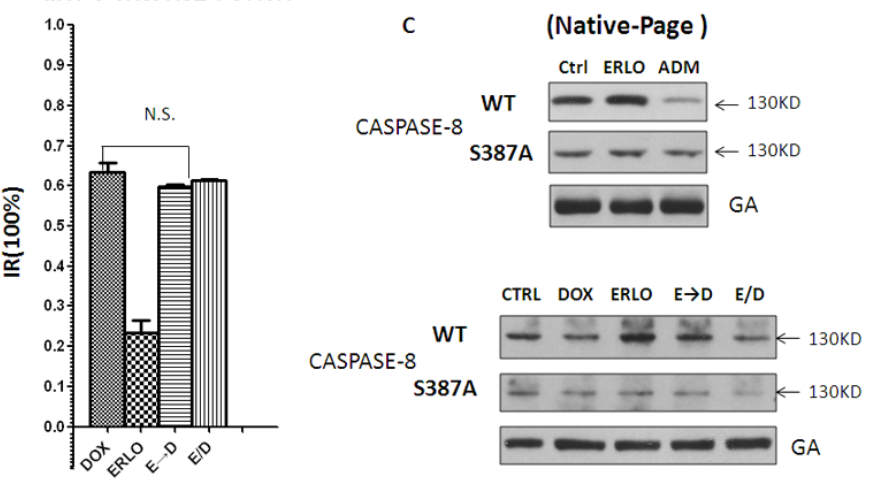

D

MCF-7 caspase-8 S387A
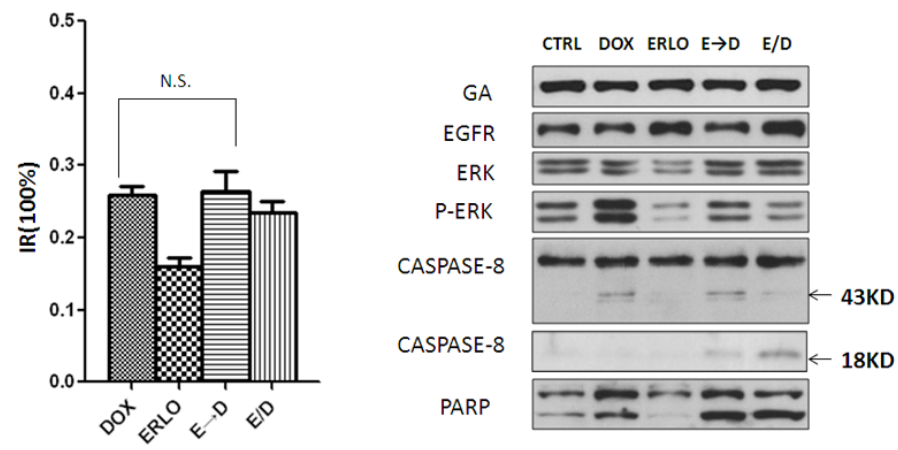
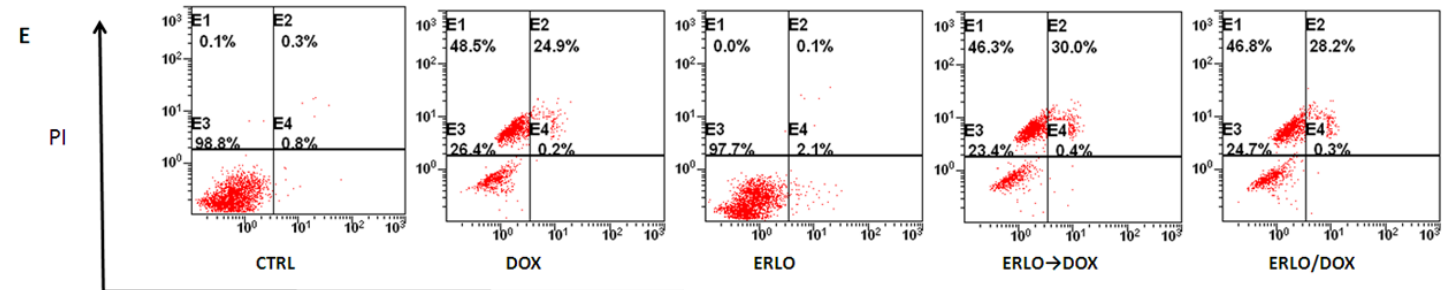

MCF-7 caspase- 8 S387A

Annexin- $V$

Figure 5: Erlotinib induces pro-caspase-8 homodimerization by attenuating the phosphorylation of pro-caspase-8 at S387 mediated by pERK 1/2. A, B. MTT assay assessing the proliferation inhibitory in MCF-7 and MDA-MB-468 cells transfeted with S387A mutant caspase-8 or WT treated with DOX, ERLO, ERLO $\rightarrow$ DOX and ERLO/DOX. MTT assay was made $12 \mathrm{~h}$ after the addition of doxorubicin. C. Native-page Western blot analysis of caspase- 8 dimerization in the DOX, ERLO, ERLO $\rightarrow$ DOX and ERLO/ DOX treated cells with or without S387A mutant pro-caspase-8 transfected. Cell lysates were collected $6 \mathrm{~h}$ after the addition of doxorubicin. D. Immunoblot analysis of indicated proteins in the DOX, ERLO, ERLO $\rightarrow$ DOX and ERLO/DOX treated cells with S387A mutant procaspase-8 transfection. Cell lysates were collected $6 \mathrm{~h}$ after the addition of doxorubicin. E. Apoptosis analysing by Annexin V-FITC/PI staining in pro-caspase-8 S387A mutant MCF-7 cells treated with erlotinib and doxorubicin or sequential application. 
in treatment response and chemoresistance has been recognized [32]. The canonical MAPK signaling cascade, depending on the extracellular signal-regulated kinases (ERK), can provide a protective effect, thereby limiting DNA damage [33]. P-ERK is known to protect cancer cells from undergoing death receptor-mediated apoptosis by phosphorylating pro-caspase-8 [28]. However, its underlying molecular mechanism has not been previously elucidated.

In this study, we provide evidence for a mechanism underlying the synergy between pre-treatment with EGFR inhibitors followed by DNA-damaging chemotherapy application in breast cancer cell lines. Upon DNA damage, cancer cells undergo defensive biological behavior tactics that promote cell survival and facilitate DNA repair, for instance, by activating several known survival pathways. We found that treatment with doxorubicin resulted in P-ERK activation and ultimately led to chemoresistance in breast cancer cells. Activated ERK inhibited caspase8 -induced apoptosis by preventing pro-caspase- 8 homodimerization. Since EGFR inhibitors can reduce P-ERK levels, DNA-damaging agents in simultaneous combination with EGFR inhibitors should provide a synergistic effect. However, the co-administration of these two classes of anti-cancer agents shows no enhanced efficacy over DNA-damaging agents alone. Interestingly, our data demonstrate that pre-treatment with EGFR inhibitors can sensitize breast cancer cells to DNAdamaging agents.

Our study showed that although ERK can be inhibited through simultaneous co-administration of these two kinds of anti-cancer agents, the formation of pro-caspase- 8 homodimer was not increased, whereas pre-treatment with erlotinib could increase levels of this critical dimer. Our data suggest that pre-treatment with erlotinib can increase the pro-caspase- 8 homodimer and thereby sensitize cancer cells to strong apoptotic stimuli, such as doxorubicin.

Ras family proteins are essential downstream components of the EGFR/Ras/MAPK signaling pathways and Ras mutations are frequently detected in several cancers [34], thereby limiting the clinical benefit of EGFR inhibitors. In these Ras mutant cell lines, sequential application of EGFR inhibitors and doxorubicin may not demonstrate a superior, synergistic effect over their concurrent co-administration. However, our experiments suggest that replacing the EGFR inhibitors with more downstream inhibition via MEK-targeted therapy may also produce the same therapeutic effect.

In the past decades, therapeutic strategies for cancer have begun to shift from the use of individual agents to combined therapeutic regimens, and it is wellestablished that cytotoxic chemotherapies are most effective and can produce synergistic effects when given in combination. However, these strategies are limited by the highly complex signaling networks that enable cancer cell populations to evolve towards drug-resistant clones. Thus, identification of effective combinatorial therapeutic strategies will be vital. Our study highlights one potential synergistic mechanism underlying the superior efficacy of sequential scheduling of TKIs and cytotoxic compounds compared to concurrent co-administration and provides a rationale for designing novel combination therapies.

\section{MATERIALS AND METHODS}

\section{Cell culture and reagents}

Human breast cancer cells (MCF-7, MDA-MB-468) were cultured in DMEM medium supplemented with 10\% fetal bovine serum in a humidified atmosphere containing $5 \% \mathrm{CO} 2$ at $37^{\circ} \mathrm{C}$. Erlotinib, Lapatinib, Doxorubicin, U0126 and AZD6244 were purchased from Selleck Chemicals. Human normal IgG was obtained from Roche (Basel, Basel-Stadt, Switzerland). GAPDH antibody was obtained from Boster Biological Technology (Wuhan, China). MTT was purchased from Sigma-Aldrich (St. Louis, Missouri), and phospho-ERK, ERK antibodies were from Santa Cruz Biotechnology (Indian Gulch, California). All other antibodies were purchased from Cell Signaling Technology (Beverly, Massachusetts). The Annexin V-FITC Apoptosis Detection Kit was from BD Sciences.

\section{Cell viability/proliferation}

Cells were seeded in 96-well-plate. Metabolic viability was determined using MTT(Sigma) assay according to the manufacturer's protocol.

\section{Plasmid construction and tTransfection}

The human pcDNA3-CASP8 vector was from Addgene (Plasmid 11817). pcDNA3-CASP8 vector was constructed into another destination vector or add a different epitope tag (Flag or Ha) of choice. Sitedirected mutagenesis of pcDNA3-CASP8 was performed using QuikChange XL (Stratagene). Plasmid transient transfection was performed using Lipofectamine 2000 according to the manufacturer's instructions (Invitrogen).

\section{RNA interference}

siRNA-pro-Caspase-8 and siRNA CASPASE-9 was produced by GenePharma Target Sequences for siRNA: CASPASE-8 sense $\quad\left(5^{\prime}-3^{\prime}\right)$

GAUACUGUCUGAUCAUCAAtt, antisense $\left(5^{\prime}-3^{\prime}\right)$

UUGAUGAUCAGACAGUAUCcc; CASPASE-9 sense 
(5'-3') : GGUGCUCAGACCAGAGAUUTT, antisense (5'-3') : AAUCUCUGGUCUGAGCACCTT; commercial siRNA-ERK 1/2 was from Cell Signaling (6560); All siRNAs were transfected using Lipofectamine ${ }^{\circledR}$ RNAiMAX Transfection Reagent (life technologies) according to the manufacturer's protocol.

\section{Western blot analysis and immunoprecipitation}

Whole cell extracts were generated by direct lysis with $1 \times$ Cell Lysis Buffer (Cell signaling technology, \#9873) adding $1 \mathrm{mM}$ PMSF immediately before use. Samples were boiled by addition $6 \times$ SDS sample buffer for $10 \mathrm{~min}$ at $100^{\circ} \mathrm{C}$ and resolved by SDS-PAGE. For immunoprecipitation, cells were lysed by E1A lysis buffer [250 mM NaCl, $50 \mathrm{mM}$ HEPES (pH 7.5), 0.1\% NP-40, 5 mM EDTA, protease inhibitor cocktail (Roche)]. Immunoprecipitation was carried out either by incubating FLAG beads at $4^{\circ} \mathrm{C}$ with lysate overnight or by incubate appropriate antibody with cell lysate for 2-3 hours, followed by incubating Protein-A/G beads overnight (Roche). Immunoprecipitates were washed 3 times with cold lysis buffer and eluted with SDS loading buffer by boiling for $10 \mathrm{~min}$.

\section{Native-page western blot analysis}

For Native-PAGE, cell extracts were generated by direct lysis with $1 \times$ Cell Lysis Buffer (Cell signaling technology, \#9873) adding $1 \mathrm{mM}$ PMSF immediately before use. Cells were disrupted on ice (20minutes) and obtained by centrifugation $\left(13000 \times \mathrm{g}\right.$ for $15 \mathrm{~min}$ at $\left.4^{\circ} \mathrm{C}\right)$, then added $6 \times$ loading buffer. Proteins were separated in a $8 \%$ polyacrylamide gel using a tris-glycine running buffer excluding sodium dodecyl sulphate (SDS) at $4^{\circ} \mathrm{C}$. After transfer to a PVDF membrane, the membrane was blocked with $5 \%$ milk in TBST containing $0.05 \%$ Tween 20 (PBST) for $2 \mathrm{~h}$, it was probed with various primary antibodies overnight at $4^{\circ} \mathrm{C}$, followed by incubation with HRP-conjugated secondary antibodies for at least $2 \mathrm{~h}$ at room temperature, then washed in TBST and visualized with enhanced chemiluminescence reagent following the manufacturer's instructions (Thermo Fisher Scientific Inc). Immunoreactivity was detected using the Amersham ECL Prime Western Blot detection reagent (GE Healthcare, Fairfield, CT, USA) according to the manufacturer's instructions.

\section{Annexin V-FITC/PI staining}

According to the protocol of The Annexin V-FITC Apoptosis Detection Kit(BD Sciences), Cells with different treatments were resuspended in $100 \mu 11 \times$ Binding Buffer at a concentration of $\sim 1 \times 10^{6}$ cells $/ \mathrm{ml}$. Add $5 \mu \mathrm{l}$
FITC Annexin V-FITC and $5 \mu \mathrm{l}$ PI, cells were incubated at room temperature for $15 \mathrm{~min}$ in the dark. Add $400 \mu \mathrm{l}$ of $1 \times$ Binding Buffer to each tube. Apoptosis was analysed by flow cytometry (BD Company, USA) at the wavelength of 488nm immediately(within $1 \mathrm{hr}$ ).

\section{Statistical analysis}

Data are presented as the mean \pm SEM unless otherwise stated. Student's t test was used to compare two groups for statistical significance analysis.

\section{ACKNOWLEDGMENTS}

This study was supported by Major Science and Technology Project of the National Basic Research Program (973 Program) of China (2012CB967004), Nature Science Foundation of China (81272199), Natural Science Foundation of Guangdong in China (2014A030313010).

\section{DISCLOSURE OF POTENTIAL CONFLICTS OF INTEREST}

The authors disclose no potential conflicts of interest.

\section{REFERENCES}

1. Reed JC. Apoptosis-targeted therapies for cancer. Cancer Cell. 2003; 3:17-22.

2. Williams GT. Programmed cell death: apoptosis and oncogenesis. Cell. 1991; 65:1097-1098.

3. Guo JY, Xia B and White E. Autophagy-mediated tumor promotion. Cell. 2013; 155:1216-1219.

4. Schmukler E, Kloog Y and Pinkas-Kramarski R. Ras and autophagy in cancer development and therapy. Oncotarget. 2014; 5:577-586.

5. Meier P and Vousden KH. Lucifer's labyrinth--ten years of path finding in cell death. Mol Cell. 2007; 28:746-754.

6. Vandenabeele $\mathrm{P}$, Galluzzi L, Vanden Berghe $\mathrm{T}$ and Kroemer G. Molecular mechanisms of necroptosis: an ordered cellular explosion. Nat Rev Mol Cell Biol. 2010; 11:700-714.

7. Singh A, Sweeney MF, Yu M, Burger A, Greninger P, Benes C, Haber DA and Settleman J. TAK1 inhibition promotes apoptosis in KRAS-dependent colon cancers. Cell. 2012; 148:639-650.

8. Tacar O, Sriamornsak P and Dass CR. Doxorubicin: an update on anticancer molecular action, toxicity and novel drug delivery systems. J Pharm Pharmacol. 2013; 65:157170 .

9. Minotti G, Menna P, Salvatorelli E, Cairo G and Gianni 
L. Anthracyclines: molecular advances and pharmacologic developments in antitumor activity and cardiotoxicity. Pharmacol Rev. 2004; 56:185-229.

10. Zoli W, Ulivi P, Tesei A, Fabbri F, Rosetti M, Maltoni R, Giunchi DC, Ricotti L, Brigliadori G, Vannini I and Amadori D. Addition of 5-fluorouracil to doxorubicinpaclitaxel sequence increases caspase-dependent apoptosis in breast cancer cell lines. Breast Cancer Res. 2005; 7:R681-689.

11. Hotchkiss RS, Strasser A, McDunn JE and Swanson PE. Cell death. N Engl J Med. 2009; 361:1570-1583.

12. Zhao J, Huang Y, Liu D and Chen Y. Two hits are better than one: synergistic anticancer activity of alphahelical peptides and doxorubicin/epirubicin. Oncotarget. 2015;6:1769-78.

13. Settleman J. Predicting response to HER2 kinase inhibition. Oncotarget. 2015; 6:588-589.

14. Al-Lazikani B, Banerji U and Workman P. Combinatorial drug therapy for cancer in the post-genomic era. Nat Biotechnol. 2012; 30:679-692.

15. Falchook GS, Naing A, Wheler JJ, Tsimberidou AM, Zinner R, Hong DS, Fu S, Piha-Paul SA, Janku F, Hess KR, Bastida C and Kurzrock R. Dual EGFR inhibition in combination with anti-VEGF treatment in colorectal cancer. Oncoscience. 2014; 1:540-549.

16. Corkery B, Crown J, Clynes $\mathrm{M}$ and O'Donovan N. Epidermal growth factor receptor as a potential therapeutic target in triple-negative breast cancer. Ann Oncol. 2009; 20:862-867.

17. Carey LA, Rugo HS, Marcom PK, Mayer EL, Esteva FJ, Ma CX, Liu MC, Storniolo AM, Rimawi MF, Forero-Torres A, Wolff AC, Hobday TJ, Ivanova A, Chiu WK, Ferraro M, Burrows E, et al. TBCRC 001: randomized phase II study of cetuximab in combination with carboplatin in stage IV triple-negative breast cancer. J Clin Oncol. 2012; 30:26152623.

18. Lee MJ, Ye AS, Gardino AK, Heijink AM, Sorger PK, MacBeath G and Yaffe MB. Sequential application of anticancer drugs enhances cell death by rewiring apoptotic signaling networks. Cell. 2012; 149:780-794.

19. Gatzemeier U, Pluzanska A, Szczesna A, Kaukel E, Roubec J, De Rosa F, Milanowski J, Karnicka-Mlodkowski H, Pesek M, Serwatowski P, Ramlau R, Janaskova T, Vansteenkiste J, Strausz J, Manikhas GM and Von Pawel J. Phase III study of erlotinib in combination with cisplatin and gemcitabine in advanced non-small-cell lung cancer: the Tarceva Lung Cancer Investigation Trial. J Clin Oncol. 2007; 25:1545-1552.

20. Herbst RS, Giaccone G, Schiller JH, Natale RB, Miller V, Manegold C, Scagliotti G, Rosell R, Oliff I, Reeves JA, Wolf MK, Krebs AD, Averbuch SD, Ochs JS, Grous J, Fandi A, et al. Gefitinib in combination with paclitaxel and carboplatin in advanced non-small-cell lung cancer: a phase III trial--INTACT 2. J Clin Oncol. 2004; 22:785-794.
21. Mok TS, Wu YL, Yu CJ, Zhou C, Chen YM, Zhang L, Ignacio J, Liao M, Srimuninnimit V, Boyer MJ, Chua-Tan M, Sriuranpong V, Sudoyo AW, Jin K, Johnston M, Chui $\mathrm{W}$, et al. Randomized, placebo-controlled, phase II study of sequential erlotinib and chemotherapy as first-line treatment for advanced non-small-cell lung cancer. J Clin Oncol. 2009; 27:5080-5087.

22. Mijatovic SA, Timotijevic GS, Miljkovic DM, Radovic JM, Maksimovic-Ivanic DD, Dekanski DP and Stosic-Grujicic SD. Multiple antimelanoma potential of dry olive leaf extract. Int J Cancer. 2011; 128:1955-1965.

23. Fiandalo MV and Kyprianou N. Caspase control: protagonists of cancer cell apoptosis. Exp Oncol. 2012; 34:165-175.

24. Shi Y. Mechanisms of caspase activation and inhibition during apoptosis. Mol Cell. 2002; 9:459-470.

25. Mace PD, Riedl SJ and Salvesen GS. Caspase enzymology and activation mechanisms. Methods Enzymol. 2014; 544:161-178.

26. Zonta F, Pagano MA, Trentin L, Tibaldi E, Frezzato F, Gattazzo C, Martini V, Trimarco V, Mazzorana M, Bordin L, Semenzato G and Brunati AM. Lyn-mediated procaspase 8 dimerization blocks apoptotic signaling in B-cell chronic lymphocytic leukemia. Blood. 2014; 123:875-883.

27. Jambrina PG, Bohuszewicz O, Buchete NV, Kolch W and Rosta E. Molecular mechanisms of asymmetric RAF dimer activation. Biochem Soc Trans. 2014; 42:784-790.

28. Mandal R, Raab M, Matthess Y, Becker S, Knecht R and Strebhardt K. pERK 1/2 inhibit Caspase-8 induced apoptosis in cancer cells by phosphorylating it in a cell cycle specific manner. Mol Oncol. 2014; 8:232-249.

29. Norbury CJ and Zhivotovsky B. DNA damage-induced apoptosis. Oncogene. 2004; 23:2797-2808.

30. Villunger A, Michalak EM, Coultas L, Mullauer F, Bock G, Ausserlechner MJ, Adams JM and Strasser A. p53- and drug-induced apoptotic responses mediated by $\mathrm{BH} 3$-only proteins puma and noxa. Science. 2003; 302:1036-1038.

31. McCubrey JA, Steelman LS, Chappell WH, Abrams SL, Wong EW, Chang F, Lehmann B, Terrian DM, Milella M, Tafuri A, Stivala F, Libra M, Basecke J, Evangelisti C, Martelli AM and Franklin RA. Roles of the Raf/MEK/ERK pathway in cell growth, malignant transformation and drug resistance. Biochim Biophys Acta. 2007; 1773:1263-1284.

32. Friday BB and Adjei AA. Advances in targeting the Ras/ Raf/MEK/Erk mitogen-activated protein kinase cascade with MEK inhibitors for cancer therapy. Clin Cancer Res. 2008; 14:342-346.

33. Cossa G, Gatti L, Cassinelli G, Lanzi C, Zaffaroni N and Perego P. Modulation of sensitivity to antitumor agents by targeting the MAPK survival pathway. Curr Pharm Des. 2013; 19:883-894.

34. Samatar AA and Poulikakos PI. Targeting RAS-ERK signalling in cancer: promises and challenges. Nat Rev Drug Discov. 2014; 13:928-942. 